\title{
A review of experimental studies of hydrogen as a new therapeutic agent in emergency and critical care medicine
}

\author{
Meihua Shen ${ }^{1+}$, Hongying Zhang ${ }^{2+}$, Congjun Yu', Fan Wang ${ }^{3}$ and Xuejun Sun ${ }^{4 *}$
}

\begin{abstract}
Hydrogen is the most abundant chemical element in the Universe, but is seldom regarded as a therapeutic agent. Recent evidence has shown that hydrogen is a potent antioxidative, antiapoptotic and anti-inflammatory agent and so may have potential medical applications in cells, tissues and organs. There are several methods to administer hydrogen, such as inhalation of hydrogen gas, aerosol inhalation of a hydrogen-rich solution, drinking hydrogen dissolved in water, injecting hydrogen-rich saline (HRS) and taking a hydrogen bath. Drinking hydrogen solution (saline/pure water/other solutions saturated with hydrogen) may be more practical in daily life and more suitable for daily consumption. This review summarizes the findings of recent studies on the use of hydrogen in emergency and critical care medicine using different disease models.
\end{abstract}

Keywords: Hydrogen, Reactive oxygen species, Antioxidant, Emergency, Critical care medicine

\section{Introduction}

Hydrogen is the lightest element in the Periodic Table and the most abundant chemical substance in the Universe. Most hydrogen is employed near its production site, with the two largest uses being fossil fuel processing and ammonia production, mostly for the fertilizer market. Hydrogen is seldom regarded as an important agent in medical use, especially as a therapeutic gas. However, in July 2007 researchers from the Japan Medical University Institute of Geriatrics reported that inhaled hydrogen gas has antioxidant and antiapoptotic properties that protect the brain against ischemia-reperfusion $(\mathrm{I} / \mathrm{R})$ injury and stroke by selectively reducing hydroxyl radicals $(\cdot \mathrm{OH})$ and $\mathrm{ONOO}^{-}$in cell-free systems [1]. This study aroused interest worldwide and scientists have explored the therapeutic value of hydrogen in many disease models. Accumulating evidence suggests that hydrogen can protect various cells, tissues and organs against oxidative injury [2].

This review focuses on the findings of recent studies of the effects of hydrogen in different disease models in

\footnotetext{
*Correspondence: sunxjk@hotmail.com

${ }^{\dagger}$ Equal contributors

${ }^{4}$ Department of Diving Medicine, Faculty of Naval Medicine, Second Military Medical University, 800XiangYin Road, Shanghai 200433, PR China Full list of author information is available at the end of the article
}

emergency and critical care medicine, as shown in Figure 1 . The possible mechanisms involved in its protective effects are summarized.

\section{Review}

Hydrogen therapy in the nervous system

It was first reported in 2007 that inhaled hydrogen gas has antioxidant and antiapoptotic properties that protect the brain against $I / R$ injury and stroke. In an in vitro study, researchers demonstrated that hydrogen functions as a scavenger of $\cdot \mathrm{OH}$. Then in a neonatal hypoxia-ischemia rat model, we found that $2 \%$ hydrogen gas or HRS therapy reduced apoptosis [3,4]. However, another group has reported that $2.9 \%$ hydrogen gas therapy does not ameliorate moderate-to-severe ischemic damage in a neonatal hypoxia-ischemia rat model [5], although they did find that hydrogen gas reduced infarction and hemorrhage and improved neurologic function in a rat model of middle cerebral artery occlusion. Inhalation of hydrogen gas ameliorated intracerebral hemorrhage in mice [6], and hydrogen saline protected against brain injury from experimental subarachnoid hemorrhage [7] and spinal cord I/R injury [8]. It has been reported that HRS attenuated neuronal $I / R$ injury by preserving mitochondrial function [9]. Hong and 

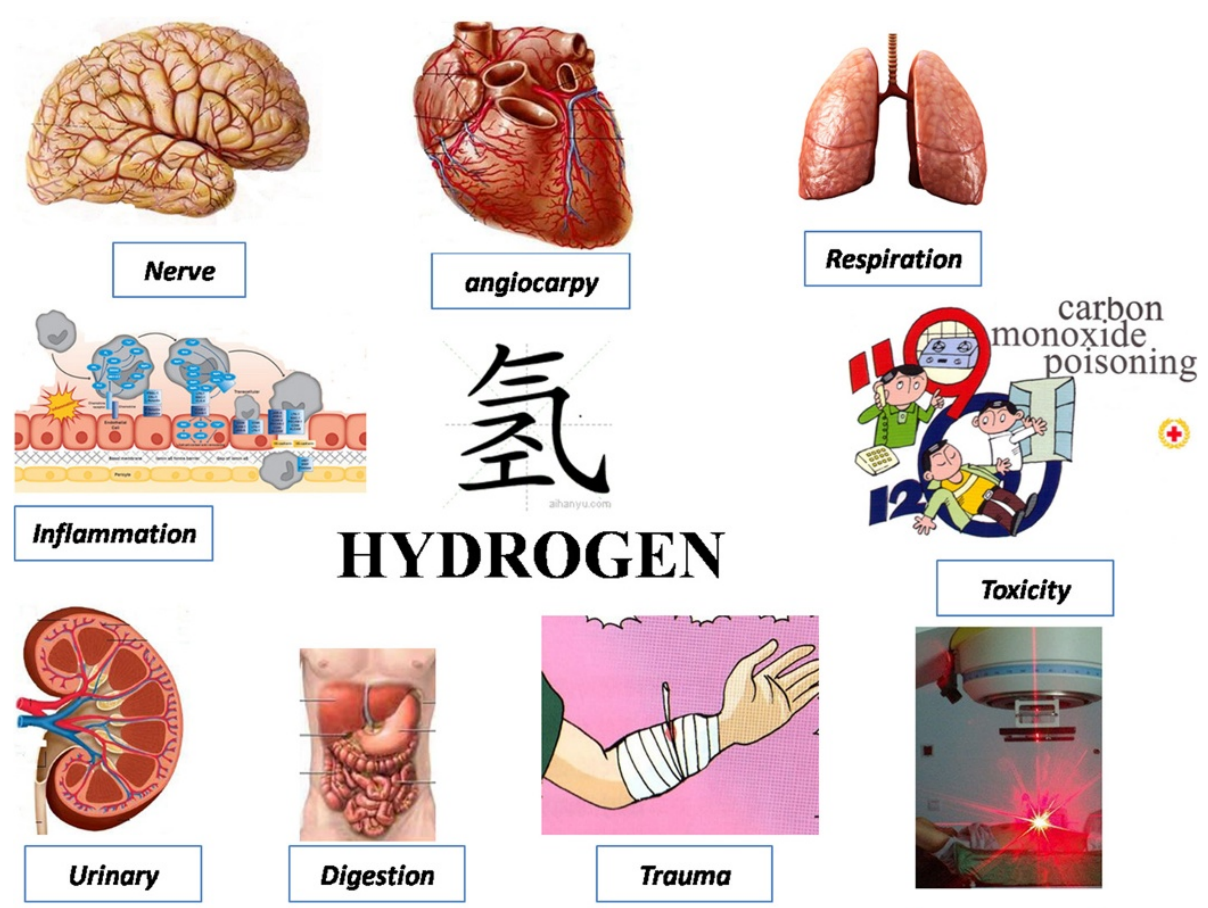

Radiation

Figure 1 Summary of potential of various preventive and therapeutic effects of hydrogen in emergency and critical care medicine using different disease models.

colleagues concluded that hydrogen can protect against neurologic damage and apoptosis early in brain injury following subarachnoid hemorrhage through the Akt/ hGSK3 $\beta$ signaling pathway [10]. Also, we found that hydrogen saline decreased 8-hydroxyl-2'-deoxyguanosine (8-OHdG), reduced malondialdehyde (MDA), interleukin-1 $\beta$ (IL-1 $\beta)$ and tumor necrosis factor alpha (TNF- $\alpha$ ) and suppressed caspase-3 activity in the ischemic brain [11]. Hyperglycemia is one of the major factors contributing to hemorrhage after ischemic stroke. Chen et al. found that the protective effect of hydrogen in the rat brain is accompanied by a reduction in oxidative stress and blood glucose levels after dextrose injection [12]. In addition, hydrogen-rich pure water has been reported to prevent superoxide formation in brain slices from vitamin $C$ depleted SMP30/GNL-knockout mice during hypoxia-re-oxygenation [13]. In a model of perinatal asphyxia in newborn pigs, ventilation with $2.1 \%$ hydrogen-supplemented room air significantly preserved cerebrovascular reactivity to hypercapnia and reduced neuronal injury induced by asphyxia-re-ventilation [14]. Interestingly, our group found that lactulose, which is used in the treatment of constipation and hepatic encephalopathy, ameliorated cerebral $I / R$ injury by inducing hydrogen [15]. Finally, it has been reported that drinking hydrogen-rich water ameliorated cognitive impairment in mice with accelerated senescence [16].

\section{Hydrogen therapy in the respiratory system}

The role of oxidative stress is well appreciated in the development of acute lung injury (ALI). Oxidative stress in ALI is believed to be initiated by products of activated lung macrophages and infiltrating neutrophils and propagates rapidly to lung epithelial and endothelial cells, leading to tissue damage and organ dysfunction. Severe burns with delayed resuscitation caused rapid lung edema and impaired oxygenation in rats, but this was ameliorated by intraperitoneal administration of HRS [17]. In a rat model of lung injury induced by intestinal I/R, HRS treatment decreased neutrophil infiltration, lipid membrane peroxidation, nuclear factor kappa B (NF-kB) activation and levels of the proinflammatory cytokines $\mathrm{IL}-1 \beta$ and TNF- $\alpha$ in lung tissues compared with saline treatment, thereby attenuating lung injury [18]. Xie and colleagues demonstrated that molecular hydrogen treatment ameliorated lipopolysaccharide (LPS)-induced ALI by reducing lung inflammation and apoptosis, which may be associated with decreased NF-kB activity [19]. Also, inhalation of hydrogen gas reduces hyperoxic lung injury in vivo [20]. Combined with fluid resuscitation, hydrogen inhalation 
attenuated lung and intestinal injury [21]. Hydrogen saline reduced airway remodeling via inactivation of $N F-\kappa B$ in a murine model of asthma [22].

\section{Hydrogen therapy in the cardiovascular system}

Emergency doctors frequently encounter acute myocardial infarction and are alert to this condition. The accelerated generation of reactive oxygen species (ROS) by reperfusion of the ischemic myocardium is a potential mediator of reperfusion injury. It has been reported that inhalation of $2 \%$ hydrogen gas rapidly increased the regional concentration of hydrogen in the area at risk of myocardial infarction before coronary blood flow was re-established in the occluded artery and alleviated I/R injury at the time of recanalization of the coronary artery [23]. Another study using the same model showed that HRS treatment attenuated regional myocardial I/R-induced cell apoptosis, as demonstrated by significant improvement in heart function parameters [24]. Furthermore, HRS decreased oxidative stress and inflammation in the area at risk in rat hearts [25]. Combination therapy with hydrogen and carbon monoxide in a syngeneic heterotopic heart transplantation model showed enhanced therapeutic efficacy via both antioxidant and anti-inflammatory mechanisms [26]. Breathing hydrogen gas plus nitric oxide reduced I/R injury in murine heart [27]. Hydrogen-containing saline protected against doxorubicin-induced heart failure [28]. There are five types of shock, among which hemorrhagic shock predominates in emergency department. Uncontrolled hemorrhagic shock can cause organ hypoperfusion, tissue ischemia and hypoxia, inflammatory cytokine release and the generation of excess oxygen-derived free radicals, all of which can result in multiple organ dysfunction. $\mathrm{Du}$ and colleagues reported that HRS protected against uncontrolled hemorrhagic shock [29]. Hydrogen can also reduce cerebral I/R injury and improve the prognosis of cardiopulmonary cerebral resuscitation after cardiac arrest [30].

\section{Hydrogen therapy in the digestive system}

Fukuda et al. found that inhalation of $2 \%$ hydrogen gas can suppress hepatic injury caused by warm I/R by reducing oxidative stress [31]. Recently, we reported protective effects of HRS on liver I/R injury due to reduced oxidative stress and high mobility group box 1 (HMGB1) release [32]. In another study, intestinal damage was detected under a microscope and assessed using the Chiu scoring system after $\mathrm{I} / \mathrm{R}$ injury. Serum diamine oxidase activity, tissue MDA and myeloperoxidase (MPO) activity, and serum TNF- $\alpha$, IL- $1 \beta$ and IL- 6 levels were all increased significantly by $\mathrm{I} / \mathrm{R}$ injury. HRS reduced these tissue injury markers and relieved the morphologic intestinal injury [33]. Simultaneously, HRS treatment significantly attenuated the severity of intestinal $I / R$ injury by inhibiting apoptosis and promoting enterocyte proliferation, limiting neutrophil infiltration and lipid oxidation, and ameliorating the decreased contractility response to potassium chloride [34]. Moreover, HRS treatment also significantly attenuates the severity of L-arginine-induced acute pancreatitis in rats by inhibiting oxidative stress, apoptosis and NF-kB activation and promoting acinar cell proliferation [35]. Sun found that acute liver injury, hepatic cirrhosis and hepatocyte proliferation in experimental liver injury were reduced by HRS through the quenching of detrimental ROS [36]. Moreover, HRS was shown to attenuate liver damage induced by obstructive jaundice or endotoxin $[37,38]$ and to protect against necrotizing enterocolitis in neonatal rats [39] and acute peritonitis [40].

\section{Hydrogen therapy in the urinary system}

Renal I/R injury, including transplantation, surgical revascularization of the renal artery, shock, partial nephrectomy and the treatment of suprarenal aortic aneurysms, is a common finding in clinical settings. Mediated by ROS, I/R is the primary cause of acute kidney injury, particularly in patients hospitalized in intensive care units. Shingu et al. reported that, after I/R injury, serum 8-OHdG levels were significantly increased; histologic analysis revealed interstitial congestion, edema, inflammation and hemorrhage in renal tissue. HRS reversed these changes and relieved mitochondrial morphologic renal injury [41]. In our study, a rat model of renal $I / R$ injury was induced by $45 \mathrm{~min}$ of occlusion of both renal pedicles followed by $24 \mathrm{~h}$ of reperfusion. We found that HRS attenuated the renal I/R injury, possibly by reduction of oxidative stress and inflammation [42]. In February 2014, it was reported that inhalation of hydrogen (2.5\%) markedly reduced levels of serum BUN, CREA and MDA, and thus could attenuate renal $\mathrm{I} / \mathrm{R}$ injury (I/R of the left renal pedicle following right nephrectomy) in rats [43].

\section{Hydrogen therapy and trauma}

It has been reported that administration of HRS reduced acute spinal cord contusion injury by decreasing the number of apoptotic cells, suppressing oxidative stress, increasing the release of brain derived neurotrophic factor and improving locomotor function [44]. Inhalation of $2 \%$ hydrogen significantly attenuated traumatic brain injury in rats [45]. Hydrogen treatment before irradiation significantly inhibited ionizing radiation-induced injury in human lymphocyte AHH-1 cells and protected the gastrointestinal endothelium of mice against radiation-induced injury [46]. Recently, it was shown that irrigation of the cornea with isotonic hydrogen solution significantly reduced angiogenesis after alkali burn injury, and hydrogen down-regulated ROS production by the cornea, 
NF- $\mathrm{kB}$ phosphorylation and levels of vascular endothelial growth factor and monocyte chemoattractant protein-1 [47]. Drinking water protects against neurodegenerative changes induced by traumatic brain injury [48].

\section{Hydrogen therapy and inflammation}

Some studies have reported the effect of hydrogen treatment in models of inflammation. First, concanavalin A (ConA)-induced liver inflammation in mice was decreased following the administration of hydrogen-producing bacteria or hydrogen water. Second, the introduction of hydrogen down-regulated the in vitro production of both TNF- $\alpha$ and interferon- $\gamma$ by ConA-stimulated spleen lymphocytes [49]. Thirdly, hydrogen-saturated water has been shown to prevent the development of dextran sodium sulfate-induced colitis in mice, this effect most likely being due to suppression by hydrogen of macrophage activation in response to luminal bacterial antigens such as LPS [50]. Furthermore, the findings of Zhen et al. indicate that HRS had an anti-inflammatory effect in both LPS-activated macrophages and paw edema models [51]. In a mouse model of systematic inflammation, hydrogen inhalation significantly improved the survival rate of septic mice in a concentration and time dependent manner [52]. Treatment of mice with $2 \%$ hydrogen had beneficial effects on sepsis and sepsis associated organ damage, as demonstrated by decreased levels of oxidative products, increased antioxidant enzyme activity and reduced levels of (HMGB1) in serum and tissue. Furthermore, $2 \%$ hydrogen treatment has been reported to protect mice against multiple organ damage in a zymosan-induced generalized model of inflammation [53]. Some studies have found that hyperoxia may be beneficial in sepsis. However, the clinical use of hyperoxia is hindered by concerns that it could exacerbate organ injury by increasing free radical formation. Xie et al. found that combination therapy with hydrogen gas and hyperoxia has enhanced therapeutic efficacy via both antioxidant and anti-inflammatory mechanisms and might potentially be a clinically feasible approach for sepsis [54]. The same team reported in 2014 that inhalation of hydrogen gas attenuates brain injury resulting from sepsis [55].

\section{Hydrogen therapy and toxicity}

Hydrogen therapy can attenuate many kinds of cell damage resulting from drugs or chemicals. The anticancer drug cisplatin is widely employed in the treatment of many types of tumor; however, its use is limited by nephrotoxicity due to oxidative stress. Japanese researchers reported that inhalation of $1 \%$ hydrogen gas or drinking hydrogen water ad libitum alleviated this toxicity by reducing oxidative stress [56]. Also, hydrogen reduced mortality and body weight loss and improved metamorphosis accompanying decreased apoptosis in the kidney, but did not impair the antitumor activity of cisplatin against cancer cell lines in vitro or in tumor-bearing mice in vivo. The protective effect of HRS against cisplatin-induced nephrotoxicity was verified in rats using dynamic contrast enhanced computed tomography [57]. In a model of antimycin A-induced auditory hair cell damage, incubation of hair cells in a hydrogen-saturated medium significantly reduced the generation of ROS and subsequent lipid peroxidation in auditory epithelia, leading to increased hair cell survival [58]. Hydrogen gas has also been reported to protect against the morphologic and functional vestibular hair cell damage induced by ROS [59]. Exposure to high concentrations of oxygen may lead to ALI. Zheng et al. [60] found that saturated hydrogen saline alleviated hyperoxia-induced pulmonary injury, which was partly responsible for the inhibition of oxidative damage. It was also found that HRS ameliorated hyperoxia-induced ALI by reducing oxidative stress and inflammatory cascades in lung tissue [61]. Increased production of ROS is crucial in the pathogenesis of carbon monoxide poisoning. In our research, we found that hydrogen provides neural protection against acute carbon monoxide [62] poisoning and reduces delayed neurologic sequelae [63].

\section{Hydrogen therapy and radiation injury}

Radiotherapy is an important modality of cancer treatment, but ionizing radiation-induced damage caused by $\cdot \mathrm{OH}$ following radiolysis of $\mathrm{H}_{2} \mathrm{O}$ is a problem and is usually initially dealt with by emergency department and intensive care unit physicians. It has been reported that HRS has a cardioprotective effect against radiation-induced injury [46]. Qian et al. [64] demonstrated that intraperitoneal injection of HRS before irradiation protected the gastrointestinal endothelia from radiation-induced injury. Radiation pneumonitis is another common complication. Chuai and colleagues reported that aerosol inhalation of a hydrogen-rich solution may be an effective and novel preventive strategy for radiation pneumonitis [65]. Hydrogen also had a radioprotective effect in vivo [66]. In addition, hydrogen preserved spermatogenesis and hematopoiesis [67] and protected against immune dysfunction [68].

\section{Methods of hydrogen intake}

There are many kinds of methods to take in hydrogen (Figure 2). The main means by which hydrogen may enter the body are breathing, drinking water, injection of saturated salt water, diffusion through the skin and endogenous hydrogen production by Escherichia coli. Hydrogen gas can be easily delivered via inhalation through a ventilator circuit, facemask or nasal cannula and is a straightforward therapeutic option. Hydrogen 


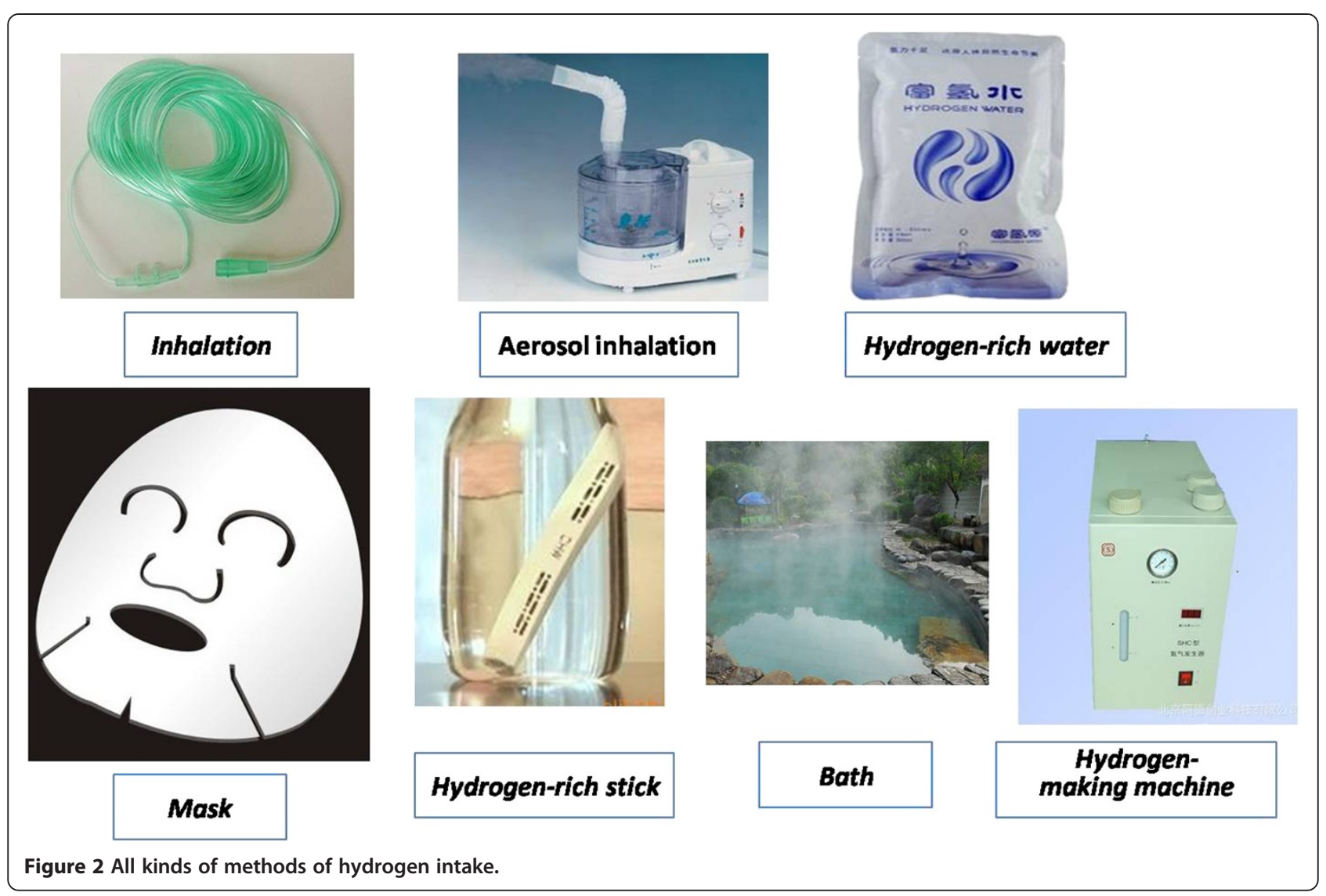

poses no risk of explosion in air or in pure oxygen when present at concentrations below $4 \%$. However, safety remains a concern and the concentration of hydrogen must be monitored and maintained at the desired level with proven, commercially available tools [69]. The safety of hydrogen for humans is demonstrated by its use in hydreliox, an exotic breathing gas mixture of $49 \%$ hydrogen, $50 \%$ helium and $1 \%$ oxygen that is used for prevention of decompression sickness and nitrogen narcosis during very deep sea diving [70]. In air, hydrogen is present at a volume fraction of $4-75 \%$ and may even reach combustible levels; however, if the temperature does not exceed $500^{\circ} \mathrm{C}$ the gas will not burn, so the risk is controllable.

Solubilized hydrogen is a portable, easily administered and safe means of delivering molecular hydrogen [71]. Drinking hydrogen-rich water has an effect comparable to that of hydrogen inhalation [56]. Hydrogen water can be made by several methods, included dissolving electrolyzed hydrogen in pure water, dissolving hydrogen in water under

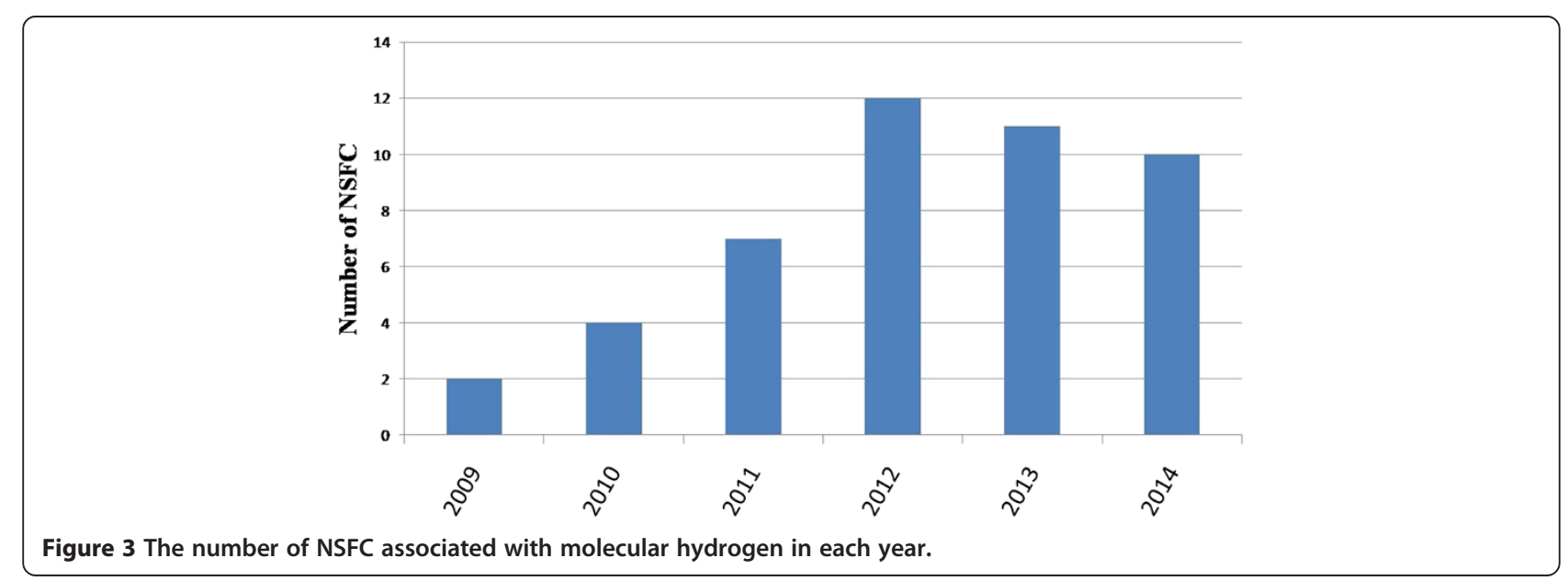


high pressure and by the use of a hydrogen gas releasing agent (magnesium reacted with water: $\mathrm{Mg}+2 \mathrm{H}_{2} \mathrm{O} \rightarrow \mathrm{Mg}$ $\left.(\mathrm{OH})_{2}+\mathrm{H}_{2}\right)$. Hydrogen water is also produced and sold commercially. Administration of hydrogen via an injectable hydrogen-rich vehicle may allow the delivery of more accurate concentrations. In the future "hydrogen bath" services will become available, and through nanoreactor engineering we may improve hydrogen release [72].

\section{Future directions}

Use of hydrogen for preventive and therapeutic purposes is a new field of investigation, for example, just in China there are 45 National Natural Science Foundation of China (NSFC) from 2009 to 2014 (Figure 3). Thus, there is limited information available on the pathways and processes that are influenced in vivo. Given the reported data, no other mechanism has been found to replace the reduction of $\mathrm{OH}$ by hydrogen. Further studies are needed to elucidate the precise mechanism and signaling pathway involved in the protective role of hydrogen as a biological molecule. It has been proposed that hydrogen may act as a gaseous signaling molecule like nitric oxide, carbon monoxide or hydrogen sulfide [73]. This new viewpoint needs further investigation. At the time of writing, only about 10 papers concerning clinical work have been published [74]. More randomized, placebo controlled trials to optimize the dose, timing and delivery of hydrogen are needed. In addition, because of its extensive and diverse effects, hydrogen seems to differ from conventional drugs that act specifically on their pharmacologic targets, the pharmacokinetics, biology and toxicity of hydrogen remains incompletely understood.

\section{Conclusions}

Hydrogen is a new potential therapeutic agent for the treatment of various diseases in emergency and critical care medicine. Although further investigations are required, with deepening of the basic theory and research into clinical applications, hydrogen may meet medical needs that at present incur considerable health burdens.

\section{Competing interests}

The authors declare that they have no competing interests.

\begin{abstract}
Authors' contributions
MHS contributed to the study design, and drafting and revision the manuscript. HYZ contributed to acquisition of data by electronic and manual search of the literature and revision of the manuscript. CJY conceived of the study and helped to draft the manuscript. FW conceived of the study, and participated in its design and coordination and helped to draft the manuscript. XJS conceived of the study, and participated in its design and coordination. All authors read and approved the final manuscript.
\end{abstract}

\section{Acknowledgments}

This work was supported by the Natural Science Foundation of Shanghai (No. 14ZR1449500), the National Nature Science Foundation of China (No. 81401855).

\section{Author details}

'Department of Emergency, Shanghai Provincial Crops Hospital, Chinese People's Armed Police Forces, 831HongXu Road, Shanghai 201103, PR China. ${ }^{2}$ Department of Quality Management, General Hospital, Chinese Armed Police Force, 69YongDing Road, Beijing 100039, PR China. ${ }^{3}$ Department of Medical Abministration, General Hospital, Chinese Armed Police Force, 69YongDing Road, Beijing 100039, PR China. ${ }^{4}$ Department of Diving Medicine, Faculty of Naval Medicine, Second Military Medical University, 800XiangYin Road, Shanghai 200433, PR China.

Received: 19 February 2014 Accepted: 28 October 2014 Published: 8 November 2014

\section{References}

1. Ohsawa I, Ishikawa M, Takahashi K, Watanabe M, Nishimaki K, Yamagata K, Katsura K, Katayama Y, Asoh S, Ohta S: Hydrogen acts as a therapeutic antioxidant by selectively reducing cytotoxic oxygen radicals. Nat Med 2007, 13:688-694.

2. Huang CS, Kawamura T, Toyoda Y, Nakao A: Recent advances in hydrogen research as a therapeutic medical gas. Free Radic Res 2010, 44:971-982.

3. Cai J, Kang Z, Liu K, Liu W, Li R, Zhang JH, Luo X, Sun X: Neuroprotective effects of hydrogen saline in neonatal hypoxia-ischemia rat model. Brain Res 2009, 1256:129-137.

4. Cai J, Kang Z, Liu WW, Luo X, Qiang S, Zhang JH, Ohta S, Sun X, Xu W, Tao H, Li R: Hydrogen therapy reduces apoptosis in neonatal hypoxia-ischemia rat model. Neurosci Lett 2008, 441:167-172.

5. Matchett GA, Fathali N, Hasegawa Y, Jadhav V, Ostrowski RP, Martin RD, Dorotta IR, Sun $\mathrm{X}$, Zhang JH: Hydrogen gas is ineffective in moderate and severe neonatal hypoxia-ischemia rat models. Brain Res 2009, 1259:90-97.

6. Manaenko A, Lekic T, Ma Q, Zhang JH, Tang J: Hydrogen inhalation ameliorated mast cell-mediated brain injury after intracerebral hemorrhage in mice. Crit Care Med 2013, 41:1266-1275.

7. Zhuang Z, Sun XJ, Zhang X, Liu HD, You WC, Ma CY, Zhu L, Zhou ML, Shi JX: Nuclear factor-KB/BCl-XL pathway is involved in the protective effect of hydrogen-rich saline on the brain following experimental subarachnoid hemorrhage in rabbits. J Neurosci Res 2013, 91:1599-1608.

8. Zhou L, Wang X, Xue W, Xie K, Huang Y, Chen H, Gong G, Zeng Y: Beneficial effects of hydrogen-rich saline against spinal cord ischemia-reperfusion injury in rabbits. Brain Res 2013, 1517:150-160.

9. Cui Y, Zhang H, Ji M, Jia M, Chen H, Yang J, Duan M: Hydrogen-rich saline attenuates neuronal ischemia-reperfusion injury by protecting mitochondrial function in rats. I Surg Res, in press.

10. Hong Y, Shao A, Wang J, Chen S, Wu H, McBride DW, Wu Q, Sun X, Zhang J: Neuroprotective effect of hydrogen-rich saline against neurologic damage and apoptosis in early brain injury following subarachnoid hemorrhage: possible role of the Akt/GSK3 $\beta$ signaling pathway. PLoS One 2014, 9:e96212.

11. Liu Y, Liu WW, Sun XJ, Li R, Sun Q, Cai J, Kang Z, Lv S, Zhang JH, Zhang W: Hydrogen saline offers neuroprotection by reducing oxidative stress in a focal cerebral ischemia-reperfusion rat model. Med Gas Res 2011, 1:15.

12. Chen CH, Manaenko A, Zhan Y, Xu S, Xia C, Shi X, Zhang JH, Yuan H, Sun X: Hydrogen gas reduced acute hyperglycemia-enhanced hemorrhagic transformation in a focal ischemia rat model. Neuroscience 2010, 169:402-414.

13. Sato $Y$, Kajiyama S, Amano A, Kondo Y, Sasaki T, Handa S, Takahashi R, Fukui M, Hasegawa G, Nakamura N, Fujinawa H, Mori T, Ohta M, Obayashi H, Maruyama N, Ishigami A: Hydrogen-rich pure water prevents superoxide formation in brain slices of vitamin C-depleted smp30/gnl knockout mice. Biochem Biophys Res Commun 2008, 375:346-350.

14. Domoki F, Olah O, Zimmermann A, Nemeth I, Toth-Szuki V, Hugyecz M, Temesvari P, Bari F: Hydrogen is neuroprotective and preserves cerebrovascular reactivity in asphyxiated newborn pigs. Pediatr Res 2010, 68:387-392.

15. Zhai X, Chen X, Shi J, Shi D, Ye Z, Liu W, Li M, Wang Q, Kang Z, Bi H, Sun X: Lactulose ameliorates cerebral ischemia-reperfusion injury in rats by inducing hydrogen by activating Nrf2 expression. Free Radic Biol Med 2013, 65:731-741.

16. Gu Y, Huang CS, Inoue T, Yamashita T, Ishida T, Kang KM, Nakao A: Drinking hydrogen water ameliorated cognitive impairment in senescence-accelerated mice. J Clin Biochem Nutr 2010, 46:269-276. 
17. Fang Y, Fu XJ, Gu C, Xu P, Wang Y, Yu WR, Sun Q, Sun XJ, Yao M: Hydrogen-rich saline protects against acute lung injury induced by extensive burn in rat model. J Burn Care Res 2011, 32:82-91.

18. Mao YF, Zheng XF, Cai JM, You XM, Deng XM, Zhang JH, Jiang L, Sun XJ: Hydrogen-rich saline reduces lung injury induced by intestinal ischemia/ reperfusion in rats. Biochem Biophys Res Commun 2009, 381:602-605.

19. Xie K, Yu Y, Huang Y, Zheng L, Li J, Chen H, Han H, Hou L, Gong G, Wang G: Molecular hydrogen ameliorates lipopolysaccharide-induced acute lung injury in mice through reducing inflammation and apoptosis. Shock 2012, 37:548-555.

20. Kawamura T, Wakabayashi N, Shigemura N, Huang CS, Masutani K, Tanaka Y, Noda K, Peng X, Takahashi T, Billiar TR, Okumura M, Toyoda Y, Kensler TW, Nakao A: Hydrogen gas reduces hyperoxic lung injury via the Nrf2 pathway in vivo. Am J Physiol Lung Cell Mol Physiol 2013, 304:L646-656.

21. Liu W, Shan LP, Dong XS, Liu XW, Ma T, Liu Z: Combined early fluid resuscitation and hydrogen inhalation attenuates lung and intestine injury. World J Gastroenterol 2013, 19:492-502.

22. Xiao M, Zhu T, Wang T, Wen FQ: Hydrogen-rich saline reduces airway remodeling via inactivation of NF-KB in a murine model of asthma. Eur Rev Med Pharmacol Sci 2013, 17:1033-1043.

23. Hayashida K, Sano M, Ohsawa I, Shinmura K, Tamaki K, Kimura K, Endo J, Katayama T, Kawamura A, Kohsaka S, Makino S, Ohta S, Ogawa S, Fukuda K: Inhalation of hydrogen gas reduces infarct size in the rat model of myocardial ischemia-reperfusion injury. Biochem Biophys Res Commun 2008, 373:30-35.

24. Sun Q, Kang Z, Cai J, Liu W, Liu Y, Zhang JH, Denoble PJ, Tao H, Sun X Hydrogen-rich saline protects myocardium against ischemia/reperfusion injury in rats. Exp Biol Med 2009, 234:1212-1219.

25. Zhang $Y$, Sun $Q$, He B, Xiao J, Wang Z, Sun X: Anti-inflammatory effect of hydrogen-rich saline in a rat model of regional myocardial ischemia and reperfusion. Int J Cardiol 2011, 148:91-95.

26. Nakao A, Kaczorowski DJ, Wang Y, Cardinal JS, Buchholz BM, Sugimoto R, Tobita K, Lee S, Toyoda Y, Billiar TR, McCurry KR: Amelioration of rat cardiac cold ischemia /reperfusion injury with inhaled hydrogen or carbon monoxide, or both. J Heart Lung Transplant 2010, 29:544-553.

27. Shinbo T, Kokubo K, Sato Y, Hagiri S, Hataishi R, Hirose M, Kobayashi H: Breathing nitric oxide plus hydrogen gas reduces ischemia-reperfusion injury and nitrotyrosine production in murine heart. Am J Physiol Heart Circ Physiol 2013, 305:H542-550.

28. Wu S, Zhu L, Yang J, Fan Z, Dong Y, Luan R, Cai J, Fu L: Hydrogen-containing saline attenuates doxorubicin-induced heart failure in rats. Pharmazie 2014 69:633-636

29. DU Z, Jia H, Liu J, Zhao X, Wang Y, Sun X: Protective effects of hydrogen-rich saline in uncontrolled hemorrhagic shock. Exp Ther Med 2014, 7:1253-1258.

30. Huang G, Zhou J, Zhan W, Xiong Y, Hu C, Li X, Li X, Li Y, Liao X: The neuroprotective effects of intraperitoneal injection of hydrogen in rabbits with cardiac arrest. Resuscitation 2013, 84:690-695.

31. Fukuda K, Asoh S, Ishikawa M, Yamamoto Y, Ohsawa I, Ohta S: Inhalation of hydrogen gas suppresses hepatic injury caused by ischemia/reperfusion through reducing oxidative stress. Biochem Biophys Res Commun 2007, 361:670-674.

32. Liu YT, Yang LQ, Tao KM, Vizcaychipi MP, Lloyd DM, Sun X, Irwin MG, Ma D, Yu W: Protective effects of hydrogen enriched saline on liver ischemia reperfusion injury by reducing oxidative stress and HMGB1 release. BMC Gastroenterol 2014, 14:12.

33. Zheng $X$, Mao Y, Cai J, Li Y, Liu W, Sun P, Zhang JH, Sun X, Yuan H: Hydrogen-rich saline protects against intestinal ischemia/reperfusion injury in rats. Free Radic Res 2009, 43:478-484.

34. Chen H, Sun YP, Hu PF, Liu WW, Xiang HG, Li Y, Yan RL, Su N, Ruan CP, Sun XJ, Wang Q: The effects of hydrogen-rich saline on the contractile and structural changes of intestine induced by ischemia-reperfusion in rats. J Surg Res 2009, 167:316-322.

35. Chen H, Sun YP, Li Y, Liu WW, Xiang HG, Fan LY, Sun Q, Xu XY, Cai JM, Ruan CP, Su N, Yan RL, Sun XJ, Wang Q: Hydrogen-rich saline ameliorates the severity of L-arginine-induced acute pancreatitis in rats. Biochem Biophys Res Commun 2010, 393:308-313.

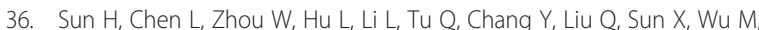
Wang $\mathrm{H}$ : The protective role of hydrogen-rich saline in experimental liver injury in mice. J Hepatol 2011, 54:471-480.

37. Liu Q, Shen WF, Sun HY, Fan DF, Nakao A, Cai JM, Yan G, Zhou WP, Shen RX, Yang JM, Sun XJ: Hydrogen-rich saline protects against liver injury in rats with obstructive jaundice. Liver Int 2010, 30:958-968.
38. Xu XF, Zhang J: Saturated hydrogen saline attenuates endotoxin-induced acute liver dysfunction in rats. Physiol Res 2013, 62:395-403.

39. Sheng Q, Lv Z, Cai W, Song H, Qian L, Wang X: Protective effects of hydrogen-rich saline on necrotizing enterocolitis in neonatal rats. J Pediatr Surg 2013, 48:1697-1706.

40. Zhang J, Wu Q, Song S, Wan Y, Zhang R, Tai M, Liu C: Effect of hydrogen-rich water on acute peritonitis of rat models. Int Immunopharmacol 2014, 21:94101.

41. Shingu C, Koga H, Hagiwara S, Matsumoto S, Goto K, Yokoi I, Noguchi T: Hydrogen-rich saline solution attenuates renal ischemia-reperfusion injury. J Anesth 2010, 24:569-574.

42. Wang F, Yu G, Liu SY, Li JB, Wang JF, Bo LL, Qian LR, Sun XJ, Deng XM: Hydrogen-rich saline protects against renal ischemia/reperfusion injury in rats. J Surg Res 2011, 167:e339-344.

43. Zeng $K$, Huang $H$, Jiang XQ, Chen XJ, Huang W: Protective effects of hydrogen on renal ischemia/reperfusion injury in rats. Sichuan Da Xue Xue Bao Yi Xue Ban 2014, 45:39-42.

44. Chen C, Chen Q, Mao Y, Xu S, Xia C, Shi X, Zhang JH, Yuan H, Sun X: Hydrogen-rich saline protects against spinal cord injury in rats. Neurochem Res 2010, 35:1111-1118

45. Ji X, Liu W, Xie K, Liu W, Qu Y, Chao X, Chen T, Zhou J, Fei Z: Beneficial effects of hydrogen gas in a rat model of traumatic brain injury via reducing oxidative stress. Brain Res 2010, 1354:196-205.

46. Qian L, Cao F, Cui J, Huang Y, Zhou X, Liu S, Cai J: Radioprotective effect of hydrogen in cultured cells and mice. Free Radic Res 2010, 44:275-282.

47. Kubota M, Shimmura S, Kubota S, Miyashita H, Kato N, Noda K, Ozawa Y, Usui T, Ishida S, Umezawa K, Kurihara T, Tsubota K: Hydrogen and $\mathrm{N}$-acetyl-Lcysteine rescue oxidative stress-induced angiogenesis in a mouse corneal alkali-burn model. Invest Ophthalmol Vis Sci 2011, 52:427-433.

48. Dohi K, Kraemer BC, Erickson MA, McMillan PJ, Kovac A, Flachbartova Z, Hansen KM, Shah GN, Sheibani N, Salameh T, Banks WA: Molecular Hydrogen in Drinking Water Protects against Neurodegenerative Changes Induced by Traumatic Brain Injury. PLoS One 2014, 9:e108034

49. Kajiya M, Sato K, Silva MJ, Ouhara K, Do PM, Shanmugam KT, Kawai T: Hydrogen from intestinal bacteria is protective for concanavalin A-induced hepatitis. Biochem Biophys Res Commun 2009, 386:316-321.

50. Kajiya M, Silva MJ, Sato K, Ouhara K, Kawai T: Hydrogen mediates suppression of colon inflammation induced by dextran sodium sulfate. Biochem Biophys Res Commun 2009, 386:11-15.

51. Xu Z, Zhu JR, Sun XJ, Zhu Z, Sun X, Jiang C: The anti-inflammation effect of hydrogen saline on paw oedema. J Inflamm 2012, 9:2.

52. Xie K, Yu Y, Pei Y, Hou L, Chen S, Xiong L, Wang G: Protective effects of hydrogen gas on murine polymicrobial sepsis via reducing oxidative stress and HMGB-1 release. Shock 2010, 34:90-97.

53. Xie K, Yu Y, Zhang Z, Liu W, Pei Y, Xiong L, Hou L, Wang G: Hydrogen gas improves survival rate and organ damage in zymosan-induced generalized inflammation model. Shock 2010, 34:495-501.

54. Xie K, Fu W, Xing W, Li A, Chen H, Han H, Yu Y, Wang G: Combination therapy with molecular hydrogen and hyperoxia in a murine model of polymicrobial sepsis. Shock 2012, 38:656-663.

55. Liu L, Xie K, Chen H, Dong X, Li Y, Yu Y, Wang G, Yu Y: Inhalation of hydrogen gas attenuates brain injury in mice with cecal ligation and puncture via inhibiting neuroinflammation, oxidative stress and neuronal apoptosis. Brain Res, in press.

56. Nakashima-Kamimura N, Mori T, Ohsawa I, Asoh S, Ohta S: Molecular hydrogen alleviates nephrotoxicity induced by an anti-cancer drug cisplatin without compromising anti-tumor activity in mice. Cancer Chemother Pharmacol 2009, 64:753-761.

57. Kitamura A, Kobayashi S, Matsushita T, Fujinawa H, Murase K: Experimental verification of protective effect of hydrogen-rich water against cisplatin-induced nephrotoxicity in rats using dynamic contrast enhanced CT. Br J Radiol 2010, 83:509-514.

58. Kikkawa YS, Nakagawa T, Horie RT, Ito J: Hydrogen protects auditory hair cells from free radicals. Neuroreport 2009, 20:689-694.

59. Taura A, Kikkawa YS, Nakagawa T, Ito J: Hydrogen protects vestibular hair cells from free radicals. Acta Otolaryngol 2010, 563(Suppl):95-100.

60. Zheng J, Liu K, Kang Z, Cai J, Liu W, Xu W, Li R, Tao H, Zhang JH, Sun X: Saturated hydrogen saline protects the lung against oxygen toxicity. Undersea Hyperb Med 2010, 37:185-192. 
61. Sun Q, Cai J, Liu S, Liu Y, Xu W, Tao H, Sun X: Hydrogen-rich saline provides protection against hyperoxic lung injury. J Surg Res 2011 165:E43-49.

62. Shen M, Cai J, Sun Q, Zhang DW, Huo ZL, He J, Sun XJ: Neuroprotective effect of hydrogen-rich saline in acute carbon monoxide poisoning. CNS Neurosci Ther 2013, 19:361-363.

63. Sun Q, Cai J, Zhou J, Tao H, Zhang JH, Zhang W, Sun XJ: Hydrogen-rich saline reduces delayed neurologic sequelae in experimental carbon monoxide toxicity. Crit Care Med 2011, 39:765-769.

64. Qian L, Cao F, Cui J, Wang Y, Huang Y, Chuai Y, Zaho L, Jiang H, Cai J: The potential cardioprotective effects of hydrogen in irradiated mice. J Radiat Res 2010, 51:741-747.

65. Chuai Y, Zhao L, Ni J, Sun D, Cui J, Li B, Qian L, Gao F, Cai J: A possible prevention strategy of radiation pneumonitis: combine radiotherapy with aerosol inhalation of hydrogen-rich solution. Med Sci Monit 2011, 17:HY1-4.

66. Yang Y, Gao F, Zhang H, Hunag Y, Zhang P, Liu C, Li B, Cai J: Molecular hydrogen protects human lymphocyte $\mathrm{AHH}-1$ cells against $12 \mathrm{C} 6+$ heavy ion radiation. Int J Radiat Biol 2013, 89:1003-1008.

67. Chuai Y, Shen J, Qian L, Wang Y, Huang Y, Gao F, Cui J, Ni J, Zhao L, Liu S, Sun X, Li B, Cai J: Hydrogen-rich saline protects spermatogenesis and hematopoiesis in irradiated BALB/c mice. Med Sci Monit 2012, 18:BR89-94.

68. Zhao S, Yang Y, Liu W, Xuan Z, Wu S, Yu S, Mei K, Huang Y, Zhang P, Cai J, $\mathrm{Ni}$ J, Zhao Y: Protective effect of hydrogen-rich saline against radiation-induced immune dysfunction. J Cell Mol Med 2014, 18:938-946.

69. Suzuki Y, Sano M, Hayashida K, Ohsawa I, Ohta S, Fukuda K: Are the effects of alpha-glucosidase inhibitors on cardiovascular events related to elevated levels of hydrogen gas in the gastrointestinal tract? FEBS Lett 2009, 583:2157-2159.

70. Abraini JH, Gardette-Chauffour MC, Martinez E, Rostain JC, Lemaire C: Psychophysiological reactions in humans during an open sea dive to $500 \mathrm{~m}$ with a hydrogen-helium-oxygen mixture. J Appl Physiol 1994, 76:1113-1118.

71. Cardinal JS, Zhan J, Wang Y, Sugimoto R, Tsung A, McCurry KR, Billiar TR, Nakao A: Oral hydrogen water prevents chronic allograft nephropathy in rats. Kidney Int 2009, 77:101-109.

72. Xia G, Chen J, Sun W, Tan Y, Guo Z, Liu H, Yu X: Well-dispersed lithium amidoborane nanoparticles through nanoreactor engineering for improved hydrogen release. Nanoscale, in press.

73. Itoh T, Hamada N, Terazawa R, Ito M, Ohno K, Ichihara M, Nozawa Y, Ito M: Molecular hydrogen inhibits lipopolysaccharide/interferon gamma-induced nitric oxide production through modulation of signal transduction in macrophages. Biochem Biophys Res Commun 2011, 411:143-149.

74. Ohta S: Molecular hydrogen as a preventive and therapeutic medical gas: initiation, development and potential of hydrogen medicine. Pharmacol Ther 2014, 144:1-11.

doi:10.1186/2045-9912-4-17

Cite this article as: Shen et al: A review of experimental studies of hydrogen as a new therapeutic agent in emergency and critical care medicine. Medical Gas Research 2014 4:17.

\section{Submit your next manuscript to BioMed Central and take full advantage of:}

- Convenient online submission

- Thorough peer review

- No space constraints or color figure charges

- Immediate publication on acceptance

- Inclusion in PubMed, CAS, Scopus and Google Scholar

- Research which is freely available for redistribution 